\title{
Implementations of Open and Closed Method Numerically: A Non-linear Equations Solution Convergence Test
}

\author{
Maulia Putri ${ }^{1}$, Syaharuddin ${ }^{2}$ \\ ${ }^{1}$ Mathematics Education Department, Islamic University of Mataram, Indonesia \\ ${ }^{2}$ Mathematics Education Department, University of Muhammadiyah Mataram, Indonesia \\ 180103014.mhs@uinmataram.ac.id, ${ }^{2}$ syaharuddin.ntb@gmail.com
}

\begin{abstract}
ABSTRAK
\section{Keyword:}

Non-linier Equations; Open-Closed Method; Numerical Method; Convergence Test.

Non-linear equations are one of the studies in mathematics. Root search in complex non-linear equations can be solved by numerical methods. Many methods to solve the equation. Therefore, the purpose of this research is to conduct simulation of closed and open methods such as Newton Raphson method, Secant method, Regula Falsi, Fixet Point, and Bisection. This is done as a form of comparative research to see the accuracy, number of iterations, and errors of each method in resolving the non-linear equations. As for the case being resolved is the roots of the exponential equation, trigonometry, logarithmic and polynomial degrees of three. The results of this study resulted in different levels of convergence in resolving each case.
\end{abstract}

\section{A. INTRODUCTION}

The non-linear equation of the system is a collection of interconnected non-linear disputes that amounts to more than one equation (Bisconti \& Franca, 2015). There are a number of purpose functions in non-linear equations that cannot be resolved analytic, but can be solved by specific methods for solving problems in non-linear equations (El-Abd, 2010). Solution of nonlinear equations in basic principles in computer science is the starting point and number of iterations (Trofimov \& Trykin, 2014), (Ratu, Negara, Mandailina, \& Sucipto, 2017). Iteration is a recurring process until an answer is found. The usual iteration techniques are used to find the roots of equations, system solutions of linear and non-linear equations, and differential equation solutions.

Furthermore, to solve the problem of non-linear equations there are many methods and algorithms that can be used, but each of the existing methods and algorithms have their own advantages and disadvantages (Negara, Syaharuddin, Kurniawati, \& Negara, 2019). One numeric method is used to resolve issues where analytic calculations cannot be used. A numerical method is a technique used to formulate mathematical issues so that they can be solved by regular count/arithmetic operations and logic operations (Mao, Wei, \& Huang, 2010). These operations are usually done sequentially (iterations) or repeatedly to obtain approximate values that are close to the actual value.

The determination of the root equation for simple non-linear functions can be easily solved by analytical methods or exact methods, e.g. for quadratic equations $\mathrm{ax}^{2}+\mathrm{bx}+\mathrm{c}=0$, can be 


\section{2 | IJECA (International Journal of Education and Curriculum Application)}

Vol. 2, No. 2, August 2019, pp. 01-06

solved using the $\mathrm{ABC}$ formula or by factoring method. For functions that are not simple or complex for example the functions $\boldsymbol{x}-\boldsymbol{e}^{-\boldsymbol{x}}=0$ or $\boldsymbol{x} \boldsymbol{e}^{-\boldsymbol{x}}+\mathbf{1}=\boldsymbol{0}$, at a glance it seems simple, but it turns out to solve this non-linear equation is a method of recursive root search, so the results obtained will contain errors and the calculation process is long and exhausting especially if done manually. The number solution obtained on a numerical method is a solution that approaches the actual value/solution approach. The solution to the numeric method will result in a difference in the actual solution, so there is a difference between the two so-called errors. This error can be minimized by selecting the appropriate method.

There are many kinds of numerical methods to complete a system of non-linear equations including Newton Raphson, Secant, Regula Falsi, Fixet Point, and Bisection methods. These methods are quite popular methods introduced in the lecturing. The Newton-Raphson method is one of the most frequently used ways in the completion method $f(x)=0$. The Newton-Raphson method uses one starting point as an initial estimate and iterates to find a solution of non-linear equations (Van Hecke, 2011). In this case, the method will fail to be used if the starting point selection gives the first derivative value zero (Dedieu, 2015). If the first derivative value is zero then iteration cannot be performed so that the predetermined starting point cannot be used. Thus, to use the Newton Raphson method need to specify a new starting point that does not provide the first derivative value zero. In the Newton-Raphson method requires a condition that must be met i.e. the function $\mathrm{f}$ must have a value of $f^{\prime}(x)$. Therefore, the alternative way to use it is to modify the Newton-Raphson method in order to find a solution equation does not use derivatives. The secant method is a method of iteration that overcomes the weakness of Newton Raphson's method by apposition with a secant line. This method requires two preliminary guesses i.e. $\mathrm{x}_{1}$ and $\mathrm{x}_{0}$. The Secant method is also called the Linear interpolation method. In the root search process, it should not be done root-pinching by the initial interval that becomes input/input of this method.

The expected outcome of the study was to know the convergence comparison in the completion of the Non-linear equation system with Newton Raphson, Secant, Regula Falsi, Fixet Point and Bisection methods using Matlab application.

\section{B. METHODS}

In this study, researchers used 5 methods namely Newton Raphson, Secant, Regula Falsi, Fixet Point and Bisection. Each method has a formulation in its completion. As for the completion algorithm of the five methods this is the same. It can be said that the five methods have the same solution algorithm, only the different formulas. Then for the implementation of each such formulation of researchers using Matlab application. The steps taken to complete the simulation are:

1. Develop each method's programming algorithm

2. Develop compute algorithms for each method

3. Draw the graph of each non-linear equation

4. Determine the starting point of each method

5. Perform computing simulations with the help of Matlab app

6. Observe each method of the simulation result consisting of the number of iterations, the root value found and the error value achieved from the specified error value that is 0.000 .

7. Perform interpretations of simulated results.

\section{RESULT AND DISCUSSION}

Researchers are using 4 questions of non-linear equations consisting of exponential, trigonometric, logarithmic and polynomial equations. Based on the steps that have been done are the chart result of each equation according to Figure 1, Figure 2, Figure 3, and Figure 4 below. 


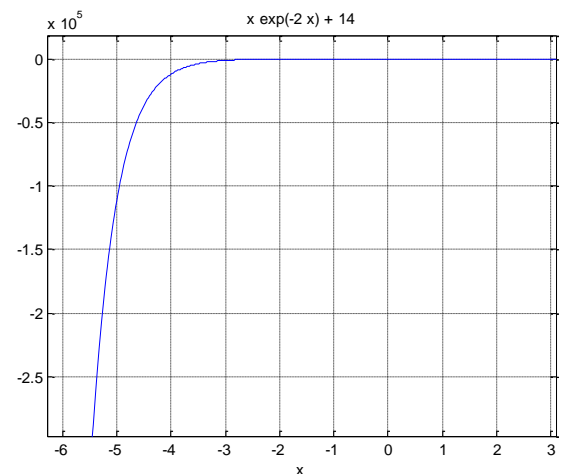

Figure 1. Graphs of Exponential Functions

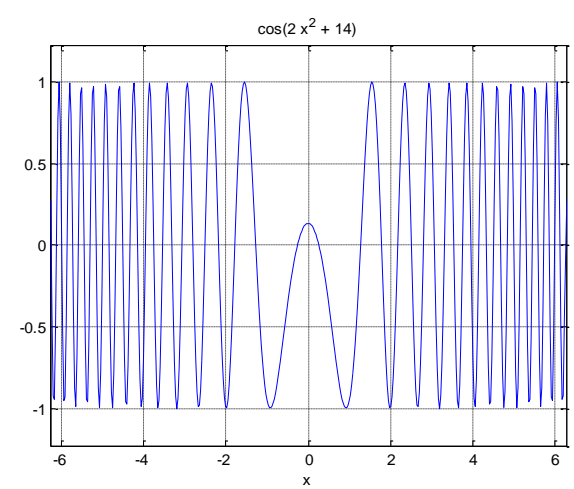

Fig. 3. Graphs of Trigonometric Functions

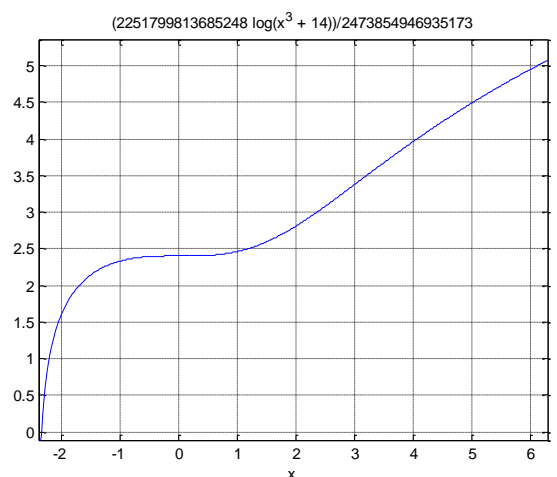

Figure 2. Graphs of Logarithmic Functions

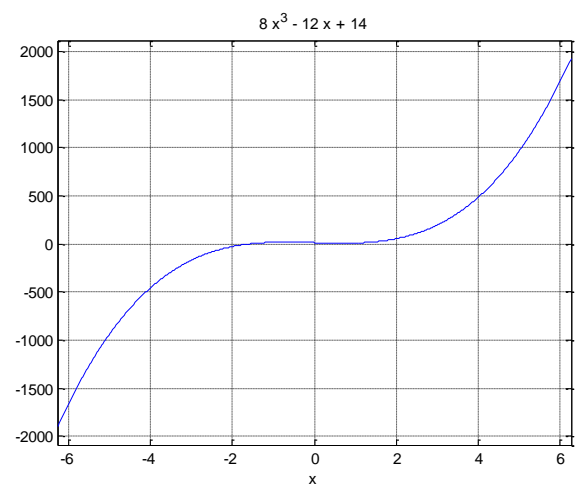

Fig. 4. Graphs of Polynomial Functions

Based on Figure 1 obtained starting point $\mathrm{x} 0=\mathrm{x} 1=-1.52$ and $\mathrm{x} 2=-1.48$ for cases of exponential equations, figure 2 for the case of polynomial degree 3 obtained $\mathrm{x} 1=-2,352$ and $\mathrm{x} 2$ $=-2,350$, Figure 3 for trigonometric cases was obtained $\mathrm{x} 1=0.27$ and $\mathrm{x} 2=-0.26$, and the last figure 4 for polynomial degree 3 cases obtained $x 1=-1.62$ and $x 2=-1.60$. Furthermore, when it determines the starting point, it is obtained the results of the computational algorithm construction of each method according to Table 1 follows.

Table 1. Formulae and Scrips Computation of Each Method

\begin{tabular}{|c|c|c|}
\hline Method & Formulae & Scrips \\
\hline Newton Raphson & $\mathrm{X}_{\mathrm{i}+1}=\mathrm{X}_{\mathrm{i}^{-}}-\frac{f(x i)}{f^{\prime}(x i)}$ & $\begin{array}{l}\mathrm{x} 2=\mathrm{x} 1- \\
(\mathrm{feval}(\mathrm{f}, \mathrm{x} 1) / \mathrm{feval}(\mathrm{f} \mathrm{diff}, \mathrm{x} 1)) ; \\
\quad \text { galat=abs }((\mathrm{x} 2-\mathrm{x} 1) / \mathrm{x} 2) ; \\
\quad \mathrm{x} 1=\mathrm{x} 2 ; \\
\mathrm{y}=\mathrm{feval}(\mathrm{f}, \mathrm{x} 1) ;\end{array}$ \\
\hline Secant & $\begin{array}{l}\mathrm{X}_{\mathrm{i}+1}=\mathrm{X}_{\mathrm{i}}- \\
\frac{x i-x i-1}{f(x i)-f(x i-1)}\end{array}$ & $\begin{array}{l}\mathrm{x} 3=\mathrm{x} 2-((\mathrm{x} 2-\mathrm{x} 1) * \mathrm{feval}(\mathrm{f}, \mathrm{x} 2) /(\text { feval } \\
(\mathrm{f}, \mathrm{x} 2)-\mathrm{feval}(\mathrm{f}, \mathrm{x} 1))) ; \\
\quad \text { galat=abs }((\mathrm{x} 3-\mathrm{x} 1) / \mathrm{x} 3) ; \\
\quad \mathrm{x} 1=\mathrm{x} 3 ; \\
\mathrm{y}=\mathrm{feval}(\mathrm{f}, \mathrm{x} 1) ;\end{array}$ \\
\hline Regula Falsi & $\begin{array}{l}\mathrm{X}_{\mathrm{i}+1}=\mathrm{x}_{\mathrm{i}}- \\
\frac{f(x i)[x i-x i-1]}{f(x i)-f(x i-1)}\end{array}$ & $\begin{array}{l}\mathrm{x} 3=\mathrm{x} 1-((\mathrm{x} 2- \\
\mathrm{x} 1) \star \mathrm{feval}(\mathrm{f}, \mathrm{x} 1) /(\mathrm{feval}(\mathrm{f}, \mathrm{x} 2)- \\
\text { feval }(\mathrm{f}, \mathrm{x} 1))) ; \\
\quad \text { galat=abs }((\mathrm{x} 3-\mathrm{x} 2) / \mathrm{x} 3) ; \\
\quad \mathrm{x} 2=\mathrm{x} 3 ; \\
\mathrm{y}=\text { feval }(\mathrm{f}, \mathrm{x} 2) ;\end{array}$ \\
\hline
\end{tabular}


4 | IJECA (International Journal of Education and Curriculum Application)

Vol. 2, No. 2, August 2019, pp. 01-06

\begin{tabular}{|c|c|c|}
\hline \multirow[t]{2}{*}{ Fixet Point } & & \multirow{2}{*}{$\begin{array}{l}\mathrm{x} 2=\text { feval }(\mathrm{g}, \mathrm{x} 1) ; \\
\quad \text { galat }=\text { abs }((\mathrm{x} 2-\mathrm{x} 1) / \mathrm{x} 2) ; \\
\quad \mathrm{x} 1=\mathrm{x} 2 ; \\
\mathrm{y}=\mathrm{feval}(\mathrm{f}, \mathrm{x} 1) ;\end{array}$} \\
\hline & $\mathrm{X}_{\text {mid }}=\frac{(x i+x i+1)}{2}$ & \\
\hline Bisection & $x_{n}=g\left(x_{n+1}\right)$ & 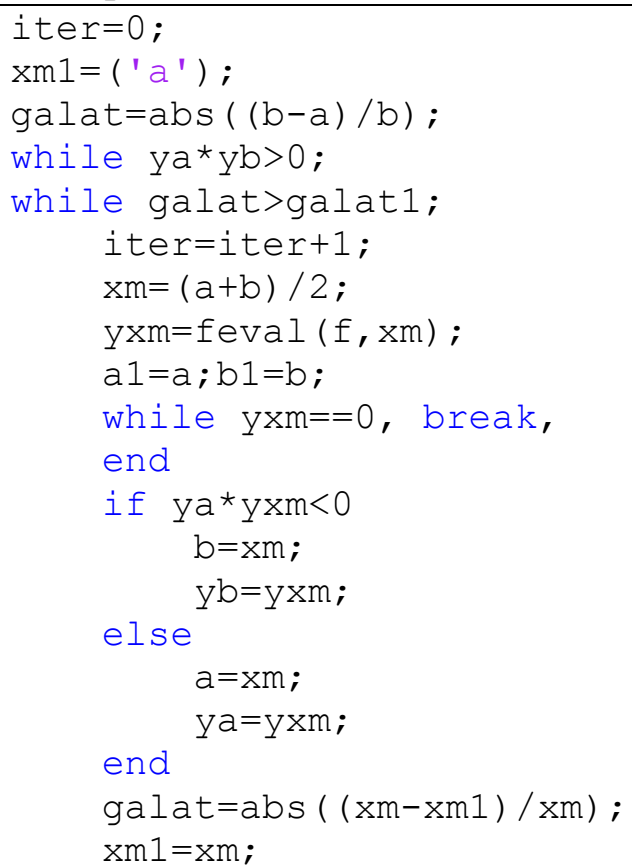 \\
\hline
\end{tabular}

Furthermore, it performs the simulation (running) of each method to see the number of iterations achieved as well as the value of the equation that is held. As for the simulation results as per Table 2, Table 3, Table 4, Table 5 follows.

Table 2. Exponential Equation Simulation Results

\begin{tabular}{clcccc}
\hline No & Method & Iterations & $\mathbf{x}_{\mathbf{i}}$ & $\mathbf{f}\left(\mathbf{x}_{\mathbf{i}}\right)$ & Error \\
\hline 1 & Newton Raphson & 4 & -1.2200732430 & -0.0000006817 & 0.000094 \\
\hline 2 & Secant & 7 & -1.2201233804 & -0.0019799693 & 0.000098 \\
\hline 3 & Regula Falsi & 8 & -1.2201082174 & -0.0013813489 & 0.000057 \\
\hline 4 & Fixet Point & 4 & 0.0711152322 & 14.0616868689 & 0.000033 \\
\hline 5 & Bisection & 9 & -1.4800781250 & -14.5669690924 & 0.000052 \\
\hline
\end{tabular}

Table 3. Trigonometric Equations Simulation Results

\begin{tabular}{clcccc}
\hline No & \multicolumn{1}{c}{ Method } & Iterations & $\mathbf{x}_{\mathbf{i}}$ & $\mathbf{f}\left(\mathbf{x}_{\mathbf{i}}\right)$ & Error \\
\hline 1 & Newton Raphson & 3 & -0.2618844604 & -0.0000000000 & 0.0000001 \\
\hline 2 & Secant & 3 & -0.2618844600 & 0.0000000004 & 0.0000003 \\
\hline 3 & Regula Falsi & 3 & -0.2618844537 & 0.0000000070 & 0.0000016 \\
\hline 4 & Fixet Point & 50 & 4.9363767760 & 0.9953742607 & 1.3571452 \\
\hline 5 & Bisection & 9 & -0.2618945312 & -0.000010549 & 0.0000745 \\
\hline
\end{tabular}

Table 4. Logarithmic Equation Simulation Results

\begin{tabular}{clcccc}
\hline No & \multicolumn{1}{c}{ Method } & Iterations & $\mathbf{x}_{\mathbf{i}}$ & $\mathbf{f}\left(\mathbf{x}_{\mathbf{i}}\right)$ & Error \\
\hline 1 & Newton Raphson & 54 & 805306569 & 628.3476 & 1.00447 \\
\hline 2 & Secant & 50 & -571.21484 & 30.09782 & 1.95940 \\
\hline 3 & Regula Falsi & 50 & -572.13797 & 30.10233 & 1.95941 \\
\hline 4 & Fixet Point & 12 & 0.13678359 & 7.310981 & 0.00006 \\
\hline 5 & Bisection & 4 & -2.3501250 & 15.07666 & 0.00005 \\
\hline
\end{tabular}


Table 5. Polynomials Equation Simulation Results

\begin{tabular}{clcccc}
\hline No & \multicolumn{1}{c}{ Method } & Iterations & $\mathbf{x}_{\mathbf{i}}$ & $\mathbf{f}\left(\mathbf{x}_{\mathbf{i}}\right)$ & Error \\
\hline 1 & Newton Raphson & 2 & -1.608679 & -0.0000003 & 0.0000606 \\
\hline 2 & Secant & 2 & -1.608679 & -0.0000254 & 0.0000473 \\
\hline 3 & Regula Falsi & 2 & -1.608678 & 0.00003283 & 0.0000465 \\
\hline 4 & Fixet Point & 7 & 0.0764137 & 13.0866043 & 0.0000482 \\
\hline 5 & Bisection & 7 & -1.608593 & 0.00428398 & 0.0000971 \\
\hline
\end{tabular}

Comparison of simulated results of each method used in resolving non-linear equations based on error values approaching and iterations used as follows:

\section{Exponential equations}

In the root lookup of the exponential equation studied, the error value closest to the specified iteration value is the Fixet Point method with error 0.0000337316 approaching the error value of 0.0001 . Here is the order of each method from the least iterations to the most iterations to find the roots in the exponential non-linear equation.

\section{Fixet Point $>>$ Bisection $>>$ Regula Falsi $>>$ Newton Raphson $>>$ Secant}

\section{Trigonometric equations}

In the root lookup in the investigated trigonometric equations, the error value that is closest to the specified error value is Newton Raphson method with error 0.0000001080 approaching the error value of 0.0001 . Here is the order of each method from the least iterations to the most iterations to find the roots on the non-linear trigonometric equations.

\section{Newton Raphson $>>$ Secant $>>$ Regula Falsi $>>$ Bisection $>>$ Fixet Point}

\section{Logarithmic equations}

In the root lookup in the researched logarithmic equation, the error value that is closest to the specified error value is the Bisection method with error 0.0000531887 approaching the error value of 0.0001 . Here is the order of each method from the least iterations to the most iterations to find the roots in the non-linear logarithmic equation.

\section{Bisection $>>$ Fixet Point $>>$ Newton Raphson $>>$ Secant $>>$ Regula Falsi}

\section{Polynomial equations}

At the root lookup in polynomial equations that are researched, the error value closest to the specified error value is the Regula Falsi method with error 0.0000465828 approaching the error value of 0.0001 . Here is the order of each method of the least iterations to the most iterations to look for roots in non-linear polynomial equations.

\section{Regula Falsi $>>$ Secant $>>$ Fixet Point $>>$ Newton Raphson $>>$ Bisection}

\section{CONCLUSION AND ADVICE}

Based on the results of research and discussion that has been displayed can be withdrawn

The conclusion that the most excellent method to solve the exponentially non-linear equation is the Fixet Point method because it has the most rapid level of conversant among the five methods, a good method to resolve Non-linear trigonometry equations is the Newton Raphson method because it has the most rapid level of conversant among the five methods, the most excellent method for solving the Non-linear logarithmic equation is the Bisection method because it has the most rapid level of conversant among the five methods, the most excellent 


\section{I IJECA (International Journal of Education and Curriculum Application)}

Vol. 2, No. 2, August 2019, pp. 01-06

method of resolving a polynomial equation is the Regula Falsi method because it has the most rapid level of convergenating among the five methods.

Suggestions for subsequent research should be developed in case of non-linear exponential equations, trigonometry, logarithm, and polynomials with different or more complicated cases and also bias applied to the case of linear equations.

\section{REFERENCES}

Bisconti, L., \& Franca, M. (2015). On a non-homogeneous and non-linear heat equation. Dynamics of Partial Differential Equations, 12(4), 289-320. https://doi.org/10.4310/DPDE.2015.v12.n4.a1

Dedieu, J.-P. (2015). Newton-Raphson Method. In Encyclopedia of Applied and Computational Mathematics (pp. 1023-1028). https://doi.org/10.1007/978-3-540-70529-1_374

El-Abd, E. M. (2010). On the existence of solutions for non-linear functional integral equation. Filomat, 24(4), 17-23. https://doi.org/10.2298/FIL1004017A

Mao, J., Wei, W., \& Huang, W. (2010). Nonlinear numerical method for stiff systems. ICCASM 2010 - 2010 International Conference on Computer Application and System Modeling, Proceedings, 6. https://doi.org/10.1109/ICCASM.2010.5620710

Negara, H. R. P., Syaharuddin, Kurniawati, K. R. A., \& Negara, H. R. P. (2019). Analysis Of Nonlinear Models For The Acceleration Of Increasing HDI In Asia. International Journal of Scientific \& Technology Research, 8(1), 60-62.

Ratu, H., Negara, P., Mandailina, V., \& Sucipto, L. (2017). Calculus Problem Solution And Simulation Using GUI Of Matlab. International Journal of Scientific \& Technology Research, 6(09), 275-279.

Trofimov, V. A., \& Trykin, E. M. (2014). Combined method for solving of $1 \mathrm{~d}$ nonlinear schrödinger equation. Lecture Notes in Electrical Engineering, 307, 173-188. https://doi.org/10.1007/978-3-319-039671_14

Van Hecke, T. (2011). On halley's correction to the newton-raphson method. Mathematical Scientist, 36(1), $37-42$. 